\title{
The Effects of Pilates Exercise Using the Three Dimensional Schroth Breathing Technique on the Physical Factors of Scoliosis Patients
}

\author{
Pil Neo HwangBo \\ Department of Physical Therapy, Daegu Health College Mailing, Daegu, Korea
}

\begin{abstract}
Purpose: This study aims to verify the effects of Pilates exercise using the three-dimensional (3D) Schroth breathing technique on scoliosis patients in comparison with the existing Pilates exercise technique, which uses lateral breathing.

Methods: The subjects were 16 scoliosis patients who went to $S$ fitness center in Daegu, Korea. They were randomly and equally divided into a Schroth Pilates exercise group (SPEG), who performed Pilates exercise using 3D Schroth breathing, and a Pilates exercise group (PEG) who performed Pilates exercise using lateral breathing. The subjects conducted the Pilates exercise three times per week for 12 weeks. Changes in their Cobb's angle, angle of trunk rotation, and chest expansion ability were measured before and after the exercise. Results: Both groups experienced significant improvement in their Cobb's angle, angle of trunk rotation, and chest expansion ability after the exercise $(p<0.05)$. Between-group comparison after the exercise showed that SPEG's improvement in Cobb's angle, angle of trunk rotation, and chest expansion ability were more significant than those of the PEG $(p<0.05)$.

Conclusion: This study verified that Pilates exercise using 3D Schroth breathing was more effective in improving scoliosis patients'physical condition than existing Pilates exercise. The researcher anticipates that the 3D Schroth breathing technique will be effectively utilized in other diverse intervention exercises besides Pilates exercise.
\end{abstract}

Keywords: Cobb's angle, Pilates exercise, Schroth 3D breathing, Schroth exercise, Scoliosis

\section{INTRODUCTION}

In modern society, a decrease in physical activity resulting from rapid economic improvement, a lack of exercise, sedentary lifestyles, and improper posture when using a smart phone may cause an imbalance in our body structure. If we do not perform exercise or receive remedial treatment, this may develop into a. During adolescence, joints become flexible and muscles and bones grow rapidly, and therefore postural distortion may easily occur. As a result, there are many adolescents with scoliosis. ${ }^{2}$ Scoliosis is defined as a lateral spinal curvature with a Cobb's angle of $10^{\circ}$ or more, characterized by the three dimensional (3D) deformity of the vertebral body, whose rotation triggers the loss of the normal curve of the sagittal plane, accompanied by the deformation of the coronal plane. ${ }^{3}$ The precise etiologies of scoliosis have not

Received Nov 14, 2018 Revised Nov 28, 2018

Accepted Dec 26, 2018

Corresponding author Pil-Neo HwangBo

E-mail pt486pn@naver.com been found. Genetic factors and behavioral characteristics related to unbalanced lifestyles are presented as relevant factors. ${ }^{4}$ Scoliosis is a 3D deformity of the spine and not only triggers noticeable changes in appearance but also disturbs the development of the thoracic cavity and the lungs and weakens the respiratory muscles, which in turn negatively affects cardiopulmonary function. Therefore, corrective and respiratory treatment for scoliosis is required. ${ }^{5}$

The goal of scoliosis treatment is for patients to reconstruct the activities of daily life with the corrected posture and enabling patients to cope with the deformity of the spine through their own efforts improves their self-esteem and was reported to increase the effects of the exercises they perform ${ }^{6,7}$ Exercise therapy as a conservative method for improving scoliosis is known to be a safe method for the treatment and prevention of scoliosis, ${ }^{8}$ and Brooks ${ }^{9}$ reported

Copylight (C2018 The Korea Society of Physical Therapy

This is an Open Access article distribute under the terms of the Creative Commons Attribution Non-commercial License (Http:// creativecommons.org/license/by-nc/4.o.) which permits unrestricted non-commercial use, distribution, and reproduction in any medium, provided the original work is properly cited. 
that Schroth exercise that applied 3D respiration for better trunk shape and respiratory ability enhanced the function of the thoracic wall and improved respiratory symptoms. Schroth's respiration method is a three-dimensional treatment that widens the concave part of the trunk of the chest and the chest area and makes the protruding part flat, ${ }^{10}$ it pulls the spinal column by itself vertically so that the muscles of the trunk are as active as possible So that the posture correction can be performed well. ${ }^{11}$ In addition, three - dimensional breathing expands the thoracic cavity when expiration and induces a significant increase in the strength of exsufflation muscles, the movement of ribs and the volume of spirometry. ${ }^{12} \mathrm{Pi}-$ lates exercise was reported to effectively ameliorate scoliosis by correcting bad posture, strengthening the muscles necessary for postural correction, and maintaining the body's balance. ${ }^{13}$ Pilates respiratory exercise was reported to influence mobility of the thoracic cavity and the spine and have positive effects on cardiovascular and respiratory variables. ${ }^{14}$ Pilates breathing is inspiration when the ribs expand sideways while the diaphragm descends, and during expiration, the diaphragm rises as the ribs gather again. At this time, it is important to extend the spine and the entire body lengthwise. ${ }^{15}$ This helps the connection between the body and the core through the diaphragmatic breathing, and breathing using the muscles and pelvic floor muscles ${ }^{16}$ Furthermore, recent studies reported that Pilates exercise had on mild scoliosis with a Cobb's angle of $20^{\circ}$ or less. ${ }^{17}$ In a study that compared the effect of Pilates exercise with that of Schroth exercise on young subjects with idiopathic scoliosis, Schroth exercise was more effective in improving their pulmonary function and inducing physical changes than Pilates exercise. ${ }^{18}$ In the Schroth exercise group using Schroth breathing, the improvement of pulmonary function as well as the Cobb's angle was reported after exercise and Pilates exercise group using Pilates breathing group had improvement compared to before exercise but less than that of Schroth group. ${ }^{18}$ Comparing differences of the two breaths, Schroth breathing differs from inspiration by expanding the concave part of scoliosis while eccentric strengthening breathing and Pilates is not.

Therefore, this study was carried out to compare the difference of effectiveness with the existing Pilates exercise when applying Schros breathing to the Pilates exercise. In addition, effective Schroth breathing method was applied to various exercises to suggest more effective exercise method for scoliosis and to increase utilization for scoliosis patients.

\section{Methods}

\section{Subjects}

This study was conducted from January to April 2018. The subjects were 16 scoliosis patients who went to at the $S$ exercise center, a specialist exercise center for scoliosis located in Daegu and whose Cobb's angle had been found to be $20^{\circ}$ or less in a prior medical check-up. Each patient was equally x-rayed at the department of radiology in Daegu, the purpose and method of this experiment were sufficiently explained to the patients, and voluntary consent to participate in this experiment was obtained from them. Those who wore an orthosis to correct scoliosis, participated in a different exercise program, had a musculoskeletal or neurological disease in addition to scoliosis, or had a mental disability were excluded from this study.

\section{Experimental methods}

\section{1) Experimental procedure}

This study randomly and equally assigned the subjects into a Schroth Pilates exercise group (SPEG), who performed Pilates exercise using Schroth breathing, and a Pilates exercise group (PEG), who performed Pilates exercise using lateral breathing. The exercise program for both groups was comprised of a warm-up exercise, a main exercise, and a cool-down exercise in both cases, the same Pilates exercise program was used with a difference in breathing methods only. ${ }^{19}$ The experiment was performed three times per week for 12 weeks, and the test results were comparatively analyzed before and after the experiment (Table 1).

During 3D Schroth breathing, the SPEG extended and raised the trunk as maximally as possible, expanded the volume of the concave region on the thoracic cavity as maximally as possible, and breathed in and then slowly breathed out, narrowing the distance

Table 1. Pilates exercise program

\begin{tabular}{ll}
\hline Pilates exercise program of both groups \\
\hline Warm up (10 min) & - Breathing \\
& - Learn posture according to the posture of the spinal column \\
$\begin{array}{ll}\text { Main Exercise } & \text { - Stretching the concave part by overball } \\
\text { (45 min) } & \text { - Single arm flot \& single shoulder shrug (by over ball) } \\
& \text { - Single elbow up } \\
& \text { - Side stretch \& mermaid } \\
& \text { - Double \& single arm push ups } \\
\text { Cool down (5 min) } & \text { - Spine segment movement \& elongation stretching }\end{array}$
\end{tabular}


between the tongue and the palate as if pronouncing "ss". While maintaining the position of the deformed part of the spine inthe direction to be corrected three-dimensionally, the subjects further expanded the thoracic cavity, with the concave part not narrowed.

During lateral breathing, the PEG extended and raised the trunk as maximally as possible, expanded the ribs aside when breathing in, with the diaphragm down and the lungs filled with air, and then breathed out pronouncing "ha" so as to gather the ribs together and raise the diaphragm.

\section{2) Measurement tools and methods}

Each subject's Cobb's angle was measured with x-ray equipment (CR 85-X, USA) using a picture archiving and communication system. X-ray imaging was performed with the subjects in a standing position. To measure the the subject's trunk, Adam's forward bend test was performed using a scoliometer (OSI, USA). ${ }^{18}$ A tape measure was employed to measure their body's ability to expand the thoracic cavity; the circumference of the chest during maximal inspiration and maximal expiration was measured. ${ }^{20}$ Their body's ability to expand the thoracic cavity was defined as the difference between the measured values during maximal inspiration and maximal expiration. All measurement procedures were taken by a physical therapist who had 10 years of clinical experience and was qualified as a Pilates instructor.

\section{Analysis method}

Using the SPSS 18.0 program, the mean and standard average of

Table 2. The general characteristics of subjects

\begin{tabular}{lcc}
\hline & SPEG $(n=8)$ & PEG $(n=8)$ \\
\hline Gender (Female) & Female $(n=8)$ & Female $(n=8)$ \\
Age (year) & $20.94 \pm 0.32^{\mathrm{a}}$ & $21.08 \pm 1.95$ \\
Weight $(\mathrm{kg})$ & $52.45 \pm 9.20$ & $53.02 \pm 8.47$ \\
Height $(\mathrm{cm})$ & $164.36 \pm 8.38$ & $163.98 \pm 9.01$ \\
Cobb's angle $\left(^{\circ}\right)$ & $18.98 \pm 4.03$ & $19.02 \pm 9.01$ \\
\hline
\end{tabular}

SPEG: Schroth breathing Pilates exercise group, PEG: Pilates exercise group. ${ }^{a}$ mean \pm SD.

Table 3. Comparison of Cobb`s angle in each group

\begin{tabular}{lcccc}
\hline & Pre-test & Post-test & Diff-value & Pre-test \\
\hline SPEG & $18.98 \pm 0.03^{\mathrm{a}}$ & $12.32 \pm 0.73$ & $6.52 \pm 0.38$ & $3.57^{*}$ \\
PEG & $19.02 \pm 9.01$ & $16.43 \pm 6.22$ & $3.69 \pm 2.25$ & $3.84^{*}$ \\
$\mathrm{t}$ & 2.74 & & $3.73^{*}$ & \\
\hline
\end{tabular}

${ }^{a}$ mean $\pm S D,{ }^{*} p<0.05$. each measured item changes in Cobb's angle, the rotational angle of the trunk, and the body's ability to expand the thoracic cavity were calculated. A paired samples t-test was conducted for within-group comparison before and after the experiment, and the independent t-test was performed for between-group comparison before and after the experiment. A statistical significance level of 0.05 was used.

\section{RESULTS}

\section{The subjects' general characteristics}

Table 2 shows the general characteristics of the subjects. There was no significant difference between the two groups in age, height, and Cobb's angle $(\mathrm{p}>0.05)$ (Table 2$)$.

\section{Comparison of Cobb's angle between the SPEG and the PEG} The Cobb's angle of both the SPEG and PEG significantly decreased after the exercise compared to before the exercise $(p<0.05)$, and in comparison between the two groups, the Cobb's angle before and after exercise showed a more significant decrease in SPEG than PEG $(\mathrm{p}<0.05)($ Table 3$)$.

\section{Comparison of the angle of trunk rotation between the SPEG and PEG}

Both groups' rotational angle of trunk significantly decreased after the exercise compared to before the exercise $(\mathrm{p}<0.05)$, and in comparison between the two groups, the rotational angle of trunk before and after exercise showed a more significant decrease in SPEG than PEG $(\mathrm{p}<0.05)($ Table 4$)$.

\section{Comparison of the chest expansion ability between the SPEG and PEG}

The ability of both the SPEG and PEG to expand the thoracic cavity significantly improved after the exercise compared to before the exercise $(\mathrm{p}<0.05)$, and in comparison between the two groups, the

Table 4. Comparison of angle of trunk rotation in each group

\begin{tabular}{llcccc}
\hline & & Pre-test & Post-test & Diff-value & Pre-test \\
\hline ATR & SPEG & $7.03 \pm 3.17^{a}$ & $3.23 \pm 1.39$ & $-3.80 \pm 1.62$ & $-3.42^{*}$ \\
& PEG & $6.98 \pm 2.09$ & $4.90 \pm 1.18$ & $-2.08 \pm 1.07$ & $-3.13^{*}$ \\
$\mathrm{t}$ & 1.02 & & $-3.09^{*}$ & \\
\hline
\end{tabular}

ATR: angle of trunk rotation.

${ }^{a}$ mean $\pm S D$, * $p<0.05$ 
Table 5. Comparison of chest expansion ability in each group

\begin{tabular}{llcccc}
\hline & & Pre-test & Post-test & Diff-value & Pre-test \\
\hline CEA & SPEG & $5.13 \pm 1.17^{a}$ & $9.83 \pm 1.39$ & $4.70 \pm 2.62$ & $4.22^{*}$ \\
& PEG & $4.93 \pm 1.09$ & $7.60 \pm 1.18$ & $2.77 \pm 2.07$ & $3.02^{*}$ \\
& $t$ & -1.33 & & $2.87^{*}$ & \\
\hline
\end{tabular}

CEA: chest expansion ability.

${ }^{a}$ mean $\pm S D,{ }^{*} p<0.05$.

chest expansion ability chest expansion ability before and after exercise showed a more significant increase in SPEG than PEG $(\mathrm{p}<0.05)$ (Table 5).

\section{DISCUSSION}

This study was conducted to verify the effects of Pilates exercise using the 3D Schroth breathing technique on scoliosis patients in comparison with existing Pilates exercise that uses lateral breathing; this was done through within-group comparison before and after the exercise and between-group comparison after the exercise.

Many studies have paid attention to electrical stimulation therapy, the use of orthosis, traction therapy, and manual therapy, as well as exercise therapy, as non-surgical, conservative treatment methods for idiopathic scoliosis. ${ }^{10,21}$ Recently, Pilates exercise using Schroth breathing was reported to be not only physically but also psychologically effective for idiopathic scoliosis patients. ${ }^{19}$

In this study, both the SPEG and the PEG saw their Cobb's angle significantly decrease after the exercise, but the SPEG's Cobb's angle decreased more significantly than the PEG's after the exercise. Araujo et al. ${ }^{22}$ asserted that Pilates exercise decreased non-structural scoliosis patients' Cobb's angle and improved their flexibility and pain. Emery et al. ${ }^{13}$ also reported that Pilates exercise for 12 weeks decreased subjects' Cobb's angle and improved their overall spine angle. Research on idiopathic scoliosis patients whose Cobb's angle was $20^{\circ}$ or more observed that Schroth Pilates exercise was significantly more effective for improving the subjects' Cobb's angle than existing Pilates exercise. ${ }^{18}$ In the present study, the SPEG who conducted Pilates exercise using Schroth breathing also saw more significant improvement in their Cobb's angle than the PEG. Therefore, effective 3D Schroth breathing is considered to increase the effect of Pilates exercise. In addition, although changes in the rotational angle of the trunk were significant in both the SPEG and the PEG, the SPEG's changes were more significant than those of the
PEG. $\operatorname{Im}^{23}$ noted that the rotational angle of the trunk in with significantly decreased after Pilates exercise, and $\mathrm{Kim}^{17}$ reported a decrease in the female college students with scoliosis after Pilates exercise. Schroth exercise that uses 3D breathing techniques was reported to effectively decrease the scoliosis patients while the thoracic rib hump was rotated in the opposite direction. ${ }^{18}$ Pilates exercise using 3D Schroth breathing is considered to provide greater improvements to the subject's rotational angle of the trunk than existing Pilates exercise.

In the present study, the body's ability to expand the thoracic cavity significantly improved in both the SPEG and the PEG, but the SPEG's ability to expand the thoracic cavity improved more significantly than that of the PEG. Yu and Lee reported the Pilates method can offer significant improvement in personal flexibility, agility, power, balance, and muscle endurance. ${ }^{24}$ Pilates exercise aimto improve flexibility of the trunk, increase the mobility of the thoracic vertebra and constrained segments, and in flat the thoracic cavity in both side directions, thereby positively affecting pulmonary functions. ${ }^{25}$ Schroth breathing techniques were reported to be conducive to recovering the functions of the diaphragm and respiratory muscles by three-dimensionally rotating the distorted thoracic cavity in the opposite direction while breathing. ${ }^{10}$ In addition, reported that the Schroth breathing exercise, which is three-dimensionally extended to the concave area compared with Pilates breathing is effective in improving the lung function of patients with scoliosis. ${ }^{18}$ In this study, eccentric expiratory muscle strengthening exercises seemed to improve the thoracic cavity expansion ability because of the breathing that extended the concave area in the Pilates exercise using 3-dimensional Schroth breathing compared to the existing Pilates breathing.

Even though both the Pilates exercise using 3D Schroth breathing and the existing Pilates exercise using lateral breathing were found to be effective for improving scoliosis patients' Cobb's angle, angle of trunk rotation, and ability to expand the thoracic cavity, the between-group comparison showed that the Pilates exercise using 3D Schroth breathing had greater effects on these factors. Accordingly, the researcher anticipates that the 3D Schroth breathing technique will be effectively utilized in other diverse intervention exercises besides Pilates exercise.

Future studies may be more effective in the generalized Pilates exercise area if applied to various subject groups and approached from 
a preventive medical perspective. In the future, it is expected that it will contribute to the improvement of scoliosis and its quality of life.

\section{REFERENCES}

1. Lee SH. The effect of Pilates on scoliosis and physical fitness in elementary school students. Dong-A University. Dissertation of Master's Degree. 2007.

2. Yu JH, Jung SM. Effects of conservative treatment on scoliosis according to early detection. J Kor Soc Phys Ther. 2010;22(4):65-71.

3. Watters H, Volansky K, Wilmarth M. The Schroth method of treatment for a patient diagnosed with scoliosis: a case report. J Nov Physiother. 2012;2:113.

4. Mooney V, Brigham A. The role of measured resistance exercises in adolescent scoliosis. Orthopedics. 2003;26(2):167-71.

5. Lonstein JE. Adolescent idiopathic scoliosis. Lancet. 1994;344(8934): 1407-12.

6. Freidel K, Petermann F, Reichel D et al. Quality of life in women with idiopathic scoliosis. Spine. 2002;27(4):87-91.

7. Weiss HR, Seibel S. Scoliosis short-term rehabilitation (SSTR)-a pilot investigation. The Internet Journal of Rehabilitation. 2009;1(1):1-8.

8. Lenssinck MLB, Frijlink AC, Berger MY et al. Effect of bracing and other conservative interventions in the treatment of idiopathic scoliosis in adolescents: a systematic review of clinical trials. Phys Ther. 2005;85(12): 1329-39.

9. Brooks WJ, Krupinski EA, Hawes MC. Reversal of childhood idiopathic scoliosis in an adult, without surgery: a case report and literature review. Scoliosis. 2009;15(4):27.

10. Lehnert-Schroth C. Three dimensional treatment for scoliosis: a physiotherapeutic method for deformities of the spine. Seoul, Yeong Mun, 2010.

11. Negrini S, Antonini G, Carabalona R. Physical exercises as a treatment for adolescent idiopathic scoliosis. A systematic review. Pediatr Rehabil. 2003;6(3-4):227-35.

12. Weiss HR. The effect of an exercise program on vital capacity and rib mobility in patients with idiopathic scoliosis. Spine.1991;16(1):88-93.

13. Emery K, Serres SJ, McMillan A et al. The effects of a Pilates training program on arm-trunk posture and movement. Clin Biomech. 2010;25(2):
124-30.

14. Owsley A. An introduction to clinical Pilates. Athl Ther Today. 2005; 10(4):19-25.

15. Moon JH. The effects of Pilates exercise on activation and thickness of abdominal superficial and deep muscles. Dankook University. Dissertation of Master's Degree. 2012.

16. Shin HJ. Effect of Pilates pelvic strength training on stress incontinence of middle-aged women. Sookmyung Women's University. Dissertation of Master's Degree. 2009.

17. Kim UC. The effect of sports massage and Pilates on the scoliosis of female collegiate dancers. Korea National Sport University. Dissertation of Doctorate Degree. 2015.

18. HwangBo PN. Comparisons of effects of Schroth exercise and Pilates exercise to female students with growing idiopathic scoliosis. Daegu University. Daegu University. Dissertation of Doctorate Degree. 2015.

19. HwangBo PN. Psychological effect of Schroth and Pilates exercise on female high school students with idiopathic scoliosis. J Kor Phys Ther. 2016:28(6):364-8.

20. Shim JH, Oh DW, Lee GW. The effects of thoracic flexibility exercise on vital capacity and chest expansion in patients with idiopathic scoliosis. Korea Academy of University Trained Physical Therapists. 2002;9(2): 145-56.

21. Park SE. The effect of Schroth treatment on Cobb's angle and pulmonary function variables of idiopathic scoliosis patients. Korea University. Dissertation of Master's Degree. 2012.

22. Araujo MEA, Silva EB, Mello DB et al. The effectiveness of the Pilates method: reducing the degree of non-structural scoliosis, and improving flexibility and pain in female college students. J Bodyw Mov Ther. 2012; 16(2):191-8.

23. Im KC. Effect of track and field exercise program or Pilates program on the Cobb's angle, physical fitness and lumbar muscle in scoliosis middle school girls. Jeju National University. Dissertation of Doctorate Degree. 2014.

24. Yu JH, Lee GC. Influence of Pilates on physical factors related to exercise performance. J Kor Phys Ther. 2011;23(3):57-63.

25. Segal NA, Hein J, Basford JR. The effects of Pilates training on flexibility and body composition: an observational study. Arch Phys Med Rehabil. 2004;85(12):1977-81. 